\title{
Relation between regional echo intensity and myocardial connective tissue in chronic left ventricular disease
}

\author{
T R D SHAW, R B LOGAN-SINCLAIR, C SURIN, R J MCANULTY, B HEARD, \\ G J LAURENT, D G GIBSON \\ From the Departments of Cardiology, Medical Electronics, Histopathology, and Thoracic Medicine (Biochemistry \\ Unit), Brompton Hospital and Cardiothoracic Institute, London
}

SUMMARY Cross sectional echocardiograms were recorded within one week of death in seven patients with valvular heart disease, four with coronary artery disesase, and nine with congenital heart disease. Regional echo amplitude was measured from the cross sectional display by constructing histograms of pixel intensity. Parietal pericardium was used as an internal standard for setting the gain of the instrument. At necropsy myocardium was taken from the free wall of the left ventricle, the papillary muscles, and the septum. Fibrosis was assessed histologically and biochemically as hydroxyproline content. In individual samples histological and biochemical estimates were correlated. In all regions other than the septum in patients with left ventricular hypertrophy, log [collagen] correlated with median pixel intensity. The amplitude of reflected echoes from the hypertrophied septum was significantly higher than that from other samples but was similarly correlated with collagen content. Agreement between echo amplitude and histological grade was significantly less good. Thus in chronic left ventricular disease myocardial collagen content appears to be the major determinant of regional echo intensity. Reproducibility of measurements and more rigorous definition of tissue abnormalities will, however, require further study.

Excessive myocardial fibrosis is an important cause of left ventricular disease. It has been correlated with clinical evidence of heart failure, ${ }^{1}$ abnormal diastolic left ventricular stiffness, ${ }^{2}$ failure of left ventricular hypertrophy to regress after valve replacement, ${ }^{3}$ and poor prognosis. ${ }^{4}$ It would thus be useful in assessing any type of left ventricular disease to be able to detect it in life. Although endomyocardial biopsy can be used, it is an invasive technique that can sample only a very small portion of the myocardium and so may give misleading values when used to assess the extent of any process that is patchy in its distribution. In the present study, we took advantage of fibrous tissue being stiffer than normal myocardium and so causing greater backscattering of ultrasound and an increase in the brightness of scarred areas on a cross sectional echocardiographic image. To do this we developed methods for the calibration, display, and

Accepted for publication 16 August 1983 quantification of regional echo amplitude using the output of a standard echocardiograph.

Myocardial fibrosis is usually detected histologically, and several studies have been performed showing an increase in association with left ventricular disease based on both biopsy ${ }^{34}$ and necropsy ${ }^{5-7}$ material. Alternatively, myocardial collagen can be detected chemically as the content of hydroxyproline containing protein. ${ }^{89}$ In the present study, of which a preliminary account has appeared elsewhere, ${ }^{10}$ we used the two approaches-histological and biochemicalto assess the extent of regional fibrosis in necropsy material and compared the results with echocardiographic observations in the same patients during life. We have thus explored $(a)$ the possibilities and limitations of extracting quantitative information from echocardiographic images obtained under clinical conditions, $(b)$ the proposition that myocardial fibrosis or collagen is a determinant of the amplitude of backscattered ultrasound, and thus of the regional amplitude of the echocardiogaphic image, and $(c)$ the 
extent to which a well recognised entity such as fibrosis can be defined in necrosy material using two independent approaches and the problems that may arise when results of these different methods are correlated in individual patients.

\section{Patients and methods}

Nineteen patients were studied. Of these, 11 were adults (aged 35-65 years; four with coronary artery disease and seven with valvular heart disease) and eight were infants or children with congenital heart disease (aged 2 days to 7 years). Of the latter, two had double outlet right ventricle, two total anomalous pulmonary venous drainage, one pulmonary atresia, one univentricular heart, one congenital mitral valve disease, and one had complete transposition and a recent Mustard operation.

\section{ECHOCARDIOGRAPHY}

In all patients, a cross sectional echocardiogram had been recorded within one week of death. Recordings were made with an ATL Mark III instrument, using a 3 or $5 \mathrm{MHz}$ transducer, as clinically appropriate. Standard parasternal, apical, and subcostal views were used when possible, but since most patients were studied immediately after open heart surgery the latter two views could not be consistently recorded to a standard adequate for quantitative analysis because of chest and pericardial drains, dressings, and the immobility of patients on respirators. Only parasternal long and short axis views, therefore, were used in the present study. As a basis for comparison, similar records were made in 15 subjects (aged 10-46 years) without evidence of severe heart disease (controls). The master gain of the instrument was adjusted so that the echo arising from the parietal pericardium in the long axis view just appeared as a continuous line at the highest level of grey scale intensity. ${ }^{11}$ Swept gain was not used, but minor changes to the image were made using the reject control. We have shown this to be without effect on measured amplitude levels. Echocardiographic images were displayed in real time, simultaneously in grey scale and after colour amplitude processing with a Brompton encoder.They were stored on $1.9 \mathrm{~cm}(3 / 4$ inch) video tape using a Sony U-matic recorder (Type VO 2631). Seven grey scale levels were displayed, the lowest being taken as 1 and appearing as cyan. Increasing levels appeared as green, yellow, red, magenta, blue, and white (level 7); black was taken as zero.

\section{Characteristics of echocardiograph}

Since the aim of the study was to make quantitative measurements from the ultrasound image rather than to rely simply on subjective judgments the perfor- mance of the echocardiograph was investigated in detail. The pulse used to excite the transducer contained a broad frequency spectrum. This was measured with the transducer connected. The components were maximum at $2 \mathrm{MHz}$, although the spectrum was almost flat between 1.5 and $5 \mathrm{MHz}$. Bandwidth limitation of the emitted acoustic pulse was imposed by the transducer itself, whose performance was determined in detail using a calibrated membrane hydrophone ${ }^{12}$ in distilled water. With moderate damping (position 5), peak positive acoustic pressure at the focus $\left(58.8 \mathrm{~mm}\right.$ ) was $0.3 \mathrm{MN} / \mathrm{m}^{2}$ (MPa), and peak negative pressure $0.29 \mathrm{MN} / \mathrm{m}^{2}$. At peak power (damping control at position 11), peak positive acoustic pressure was $3.0 \mathrm{MN} / \mathrm{m}^{2}$ and peak negative pressure $1.05 \mathrm{MN} / \mathrm{m}^{2}$. Clinical measurements were all made with settings between these two values. At higher levels, therefore, there was pronouced asymmetry of the acoustic pressure pulse due to non-linear propagation in water at high driving pressures. It is likely that similar distortion occurred in tissue, which will have served greatly to increase the harmonic content of the propagated wave.

The reception characteristics of the $3 \mathrm{MHz} 13 \mathrm{~mm}$ disc transducers were also determined in distilled water. Low level (less than $15 \mathrm{~N} / \mathrm{m}^{2}$ ) gated sine wave bursts of varying amplitude and frequency were generated by a second $10 \mathrm{MHz}$ crystal, and the resultant acoustic signal impinging on the transducer measured with a wideband membrane hydrophone interposed between the two. The ATL transducer was found to have a centre frequency not appreciably different from its nominal value of $3 \mathrm{MHz}$, and a $Q$ factor of 2 . Its electrical output was linearly related to the acoustic pressure input over a $-40 \mathrm{db}$ range, zero db corresponding to a pressure of $15 \mathrm{~N} / \mathrm{m}^{2}$. The hydrophone was also used to determine the beam shape at a number of depths. The frequency response of the RF amplifier was flat between 1.5 and $8 \mathrm{MHz}(-3 \mathrm{db}$ points). The overall gain was $106 \mathrm{db}$. The output was fullwave rectified and passed through a second order low pass filter, whose $-3 \mathrm{db}$ cutoff was $700 \mathrm{kHz}$. In the system used, the $45 \mathrm{db} \log$ compression of the demodulated signal is achieved by way of the ladder network of the flash analogue to digital converter, with three bit digitisation of the signal. For the output of the digital scan converter, a linear transfer function was used. Gamma correction was implemented on the television monitor only. The takeoff point for the video tape recorder, the encoder, and the histogram unit was at the linear output of the digital scan convertor. The possibility of phase errors at the transducer interface leading to amplitude errors was considered. These were, however, for the most part avoided by the use of full wave rectification and smoothing with a low pass $700 \mathrm{kHz}$ filter, although its use will clearly 
have been accompanied by a minor degree of information loss.

Image analysis-This was performed by a different observer from the one who had made the original recordings and was no more aware of the diagnosis than would be possible from examination of the echocardiograms themselves. End diastolic stop frames were displayed using a tape recorder with digital frame advance (Sony 5800 PS). A system of nomenclature of the left ventricle based on that of Edwards et al. ${ }^{13}$ was used. Thus the mid and basal segments of septum and free wall and the bases and tips of the papillary muscles were identified on the echocardiograms. Regional echo amplitude was assessed by counting individual picture elements (pixels). Manual and automatic counting methods were used in parallel on end diastolic frames. For the former method, frames were photographed on colour $35 \mathrm{~mm}$ film (Kodak EPT 135-36), and the transparencies projected to large size so that the individual pixels could be seen. One hundred were counted within a predetermined area and assigned to one of the seven grey scale levels. When the automatic method was used, an area of interest was localised on the stop frame video image using an interactive system under the control of the observer, and a count of the intensity of each pixel within it was performed automatically (Fig. 1). For myocardium, about 300 pixels were included; for papillary muscle, a smaller area of interest was used, which included about 100 pixels. Both methods thus allowed a histogram of regional pixel intensity to be plotted, and absolute pixel counts were derived from a digital output. Care was taken to avoid placing the area of interest over the parietal pericardial echo, which might have led to the inclusion of high amplitude echoes. Automatic pixel counts were performed on two separate occasions for each area, and these were used to derive the reproducibility of the method. Automatic and manual counting methods were also compared.

\section{NECROPSY ESTIMATION OF FIBROSIS}

Hearts were removed at necropsy and dissected after fixation in $10 \%$ formalin solution. Regions corresponding to the segments studied echocardiographically were identified, and from each two contiguous samples of $100-200 \mathrm{mg}$ were removed, one for histological and one for chemical analysis.

The extent of fibrosis was assessed histologically on sections stained with haematoxylin and eosin and

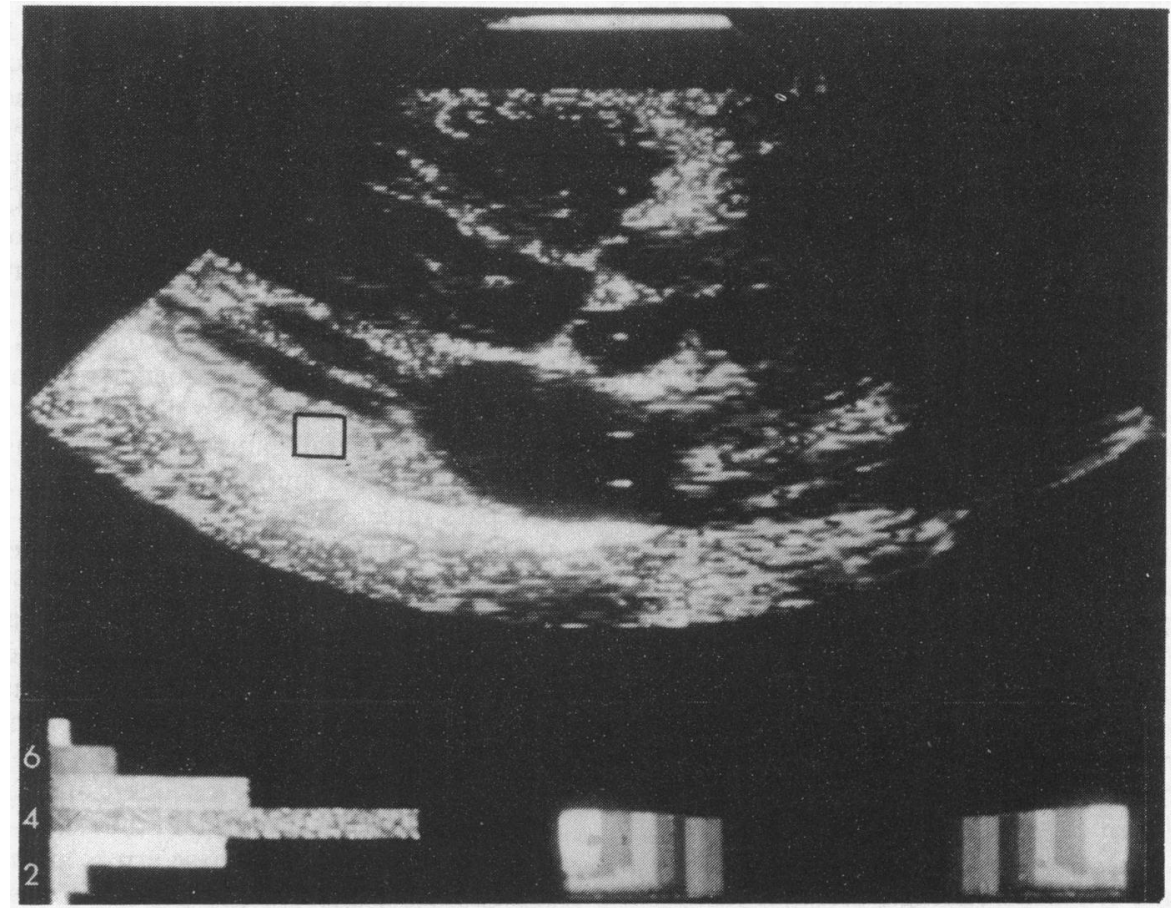

Fig. 1 Assessment of regional pixel intensity of cross sectional echocardiogram in the parasternal long axis view from a patient with ischaemic heart disease. The area of interest has been positioned over the posterior wall of the colour display. The histogram of regional pixel intensity (level scales 1-7) is shown horizontally in the lower part of the display. 
recorded as absent ( 0 ) or mild (1), moderate (2), or severe (3). Its distribution as generalised or subendocardial was also noted. These observations were made by two observers (BH and CS) who were unaware of the results of echocardiographic or biochemical analyses. Biochemical estimation of fibrosis was based on collagen content, using the method of Prockop and Udenfriend, ${ }^{14}$ with recently described modifications, ${ }^{15}$ on samples of 5-30 mg dry weight. This method is based on the detection of hydroxyproline containing protein and thus measures all collagen subtypes. The reproducibility of duplicate determinations on the same sample was less than $5 \%$. These observations were made by one of us (RM), who was unaware of the clinical diagnosis or of the results of echocardiographic or histological analyses. To assess the properties of parietal pericardium, used throughout the study as an internal standard, small samples were removed from anterior and posterior to the heart in 10 patients undergoing routine coronary artery surgery. Collagen content was estimated in the same way as for myocardial samples.

\section{DATA ANALYSIS}

Comparisons were made between median pixel intensity and collagen content, assessed chemically, for each region studied. In addition, chemical and histological estimates were compared. Since the levels of echo amplitude are based on a logarithmic rather than a linear scale median rather than mean values were used to characterise local intensity. Arithmetic means of these values were calculated between patients and standard $t$ tests used to assess differences between means. Owing to the wide spread of abnormal values of collagen content, their distribution in the sample departed significantly from normal, so that log [collagen] values were also used. Linear regression could thus be performed by the method of least squares.

\section{Results}

\section{ECHOCARDIOGRAMS}

Controls-In the controls the mean value of median pixel intensity for the posterior left ventricular wall was $0.76 \pm 0.16$ (mean \pm 1 standard deviation) in the basal region and $1.33 \pm 0.38$ at the level of the papillary muscles. The corresponding values for the septum were $1.05 \pm 0.35$ and $1.65 \pm 0.52$ respectively. For both, basal values were significantly lower than those at papillary muscle level $(p<0.01)$, and for corresponding levels septal values were higher than those from the posterior wall $(p<0.01)$.

Patients-Altogether, echocardiograms of adequate technical quality were recorded from 80 segments (4.2 per patient). Values of median pixel intensity ranged from 0.5 to 3.8 units. The standard deviation of the difference between duplicate deteminations using the automatic counting method was 0.41 grey scale level and that between manual and automatic counts for the same region was 0.58 unit.

\section{FIBROSIS}

The fibrous content varied widely between different samples. Twenty two segments in 10 patients were entirely normal histologically, while in six segments (in three patients) complete fibrous replacement had occurred. In most patients there was intermediate involvement. The fibrosis was mainly subendocardial in 28 segments and generalised in the remainder. There was macroscopic and microscopic evidence of left ventricular hypertrophy in six patients: five adults with aortic valve disease and one child with congenital mitral valve disease.

\section{COLLAGEN CONTENT}

Samples from normal adult left ventricle (controls) showed a mean collagen content of $41 \pm 16 \mu \mathrm{g} / \mathrm{mg}$ dry weight. There was no consistent difference between regions of the left ventricle. In the patients, the range was wide (between 10 and $625 \mu \mathrm{g} / \mathrm{mg}$ dry weight).

\section{COMPARISON OF METHODS}

There was moderate agreement only between collagen content assessed biochemically and fibrosis assessed histologically (Fig. 2). The regression equation was: $\log$ [collagen] $=0 \cdot 19$ [histological grade] $+1 \cdot 58$. The standard error of the estimate was 0.22 and the correlation coefficient $0.63(p<0.001)$. The standard errors of the slope and intercept were $2.81 \times 10^{-2}$ and $4 \cdot 13 \times 10^{-2}$ respectively. When a linear scale was used for collagen concentration, the corresponding value for the correlation coefficient was 0.52 . Fig. 3 shows the relation between log [collagen] and median echo

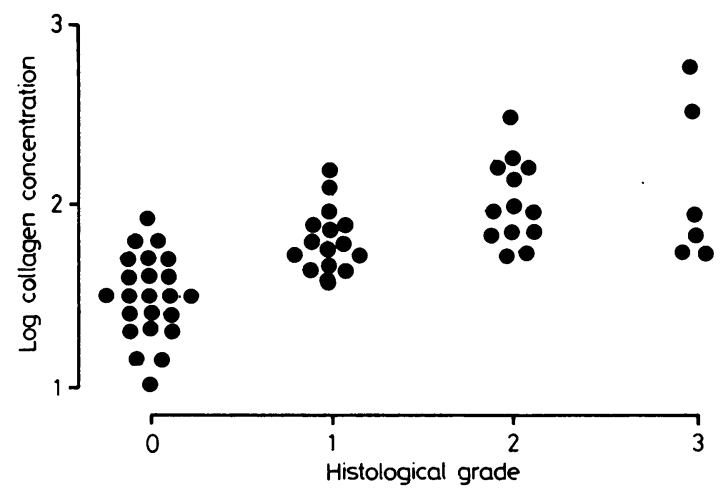

Fig. 2 Relation between estimates of regional fibrosis measured from histological grade ( 0 absent, 1 mild, 2 moderate, 3 severe) and collagen concentration. 


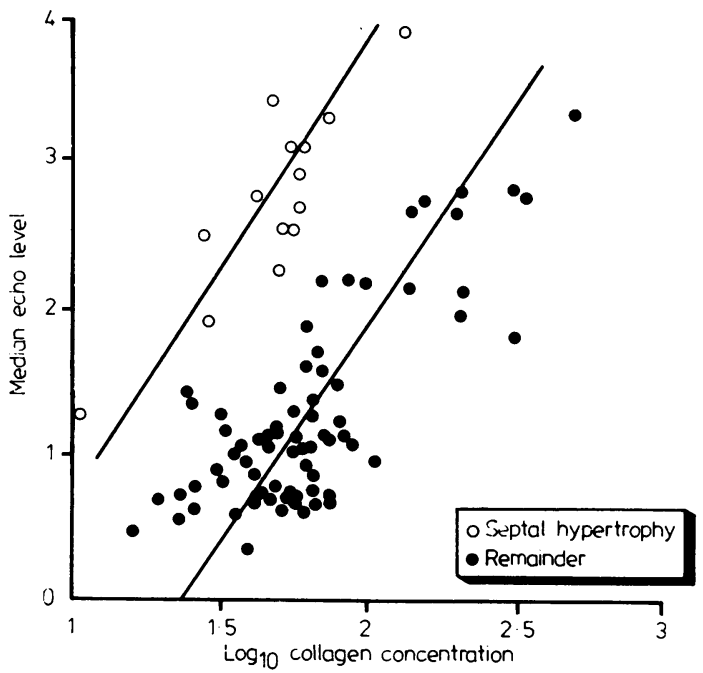

Fig. 3 Relation between regional collagen concentration assessed biochemically and median echo intensity.

intensity. Preliminary analysis showed that the points were not evenly distributed but that at any given collagen content values of median echo level from the septum of patients with left ventricular hypertrophy were significantly higher than those from the remainder of the left ventricular myocardium. In all samples but those from hypertrophied septum, the regression relation between intensity level and collagen content was: median echo level $=2.85 \log$ [collagen] $-\mathbf{3 . 8 2}$. The standard error of the estimate was 0.20 and the correlation coefficient $0.76(p<0.001)$. The standard errors of the slope and intercept were 0.12 and 0.16 respectively. When collagen concentration was expressed on a linear scale, the corresponding value for correlation coefficient was $\mathbf{0 . 7 1}$. For the values observed in the hypertrophied septum, the equation was: median echo level $=3.22 \log$ [collagen] -2.54 . The standard error of the estimate was 0.15 and of the slope and intercept 0.16 and 0.48 respectively. The correlation coefficient was $0.86(p<0.001)$. When collagen concentration was expressed on a linear scale the corresponding value was $\mathbf{0 \cdot 7 8}$. Comparison of the two relations between log [collagen] and echo intensity shows no difference in slope, but the intercept from values of patients in whom the septum was hypertrophied was significantly less negative than that from the remainder $(p<0.05)$.

Fig. 4 shows the relation between median echo intensity and histological grade. Linear regression showed some correlation between the two, the equation being: histological level $=0.46 \log$ [collagen] + 0.48 . The correlation coefficient was $0.32(p<0.02)$ and the standard error of the estimate 0.94 units. It is again apparent that at each level of fibrosis assessed histologically values of echo intensity were higher from samples derived from patients with septal hypertrophy.

\section{PERICARDIAL SAMPLES}

Two pericardial samples were available from each of 10 patients. The mean collagen content was $608 \pm 208$ $\mu \mathrm{g} / \mathrm{mg}$ dry weight. The mean value from anterior pericardium was $612 \pm 182 \mu \mathrm{g} / \mathrm{mg}$ dry weight and from posterior pericardium $605 \pm 106 \mu \mathrm{g} / \mathrm{mg}$ dry weight. These values were not significantly different. The corresponding value of median pixel intensity determined automatically in 15 controls was $6.05 \pm 0.25$ and the standard deviation of duplicate determinations $0 \cdot 18$ unit.

\section{Discussion}

Several essential conditions must be fulfilled if echocardiography is to be used to measure the amplitude as distinct from simply the position and motion of echoes returning from intracardiac structures. A digital scan converter is required rather than optical coupling. Depth compensation by swept gain cannot be used. It is also necessary to exclude all "postprocessing" procedures which alter the gamma characteristics of the instrument or use partial differentiation to "enhance" boundaries or improve the subjective qualities of the image. In the present study, therefore, the transfer function of the instrument was investigated in detail so that interrelations between

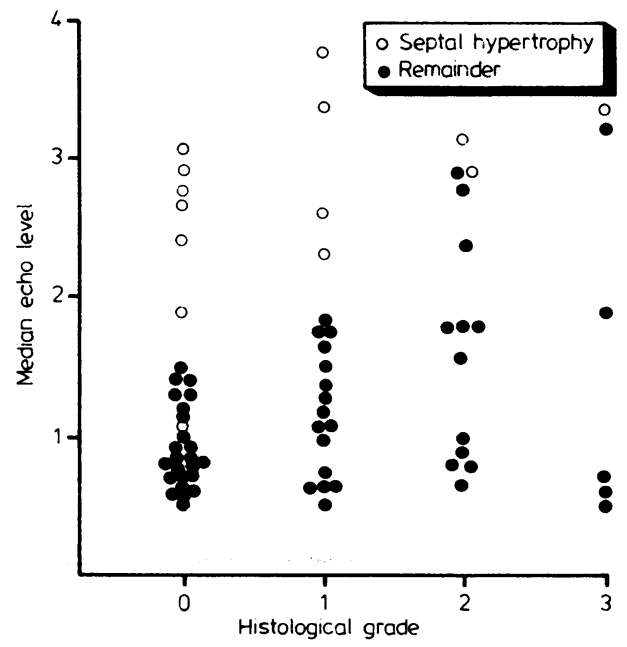

Fig. 4 Relation between regional fibrosis assessed histologically (grade 0 absent, 1 mild, 2 moderate, 3 severe) and median echo intensity. 
the pulse generated, the returning echo, and the amplitude in the final image were defined. Measurements of regional echo amplitude are likely to prove misleading unless all these factors are rigorously controlled.

Several lines of evidence have suggested that connective tissue may be an important factor in the genesis of "structural" echoes as distinct from those arising from interfaces between organs. The Young's modulus of collagen fibres is greater by a factor of 1000 than that of any tissue except bone, and in breast reflectivity is closely related to fibrosis. ${ }^{16}$ In experimental animals, Mimbs et al noted that after experimental myocardial infarction, backscattering and attenuation were closely related. ${ }^{17}$ Backscattered amplitude was considerably increased within six weeks of experimental infarction, an effect that was abolished by treatment with collagenase, although local hydroxyproline values were unaltered. Similar observations were reported in doxorubicin induced cardiomyopathy in rabbits, in which regional increases of up to $8 \mathrm{db}$ integrated backscattering could be identified after 10 to 18 weeks treatment with the drug. ${ }^{8}$ Histological examination showed vacuolisation and myocytolysis together with the development of fibrosis. In man, Rasmussen et al. ${ }^{19}$ noted an increase in the intensity of septal echoes in the M mode echocardiograms of patients with septal fibrosis confirmed at necropsy or cardiac surgery. More recently, Tanaka et al., ${ }^{20}$ using stepwise gain alterations, suggested that fibrosis was the origin of high intensity echoes originating from the myocardium. Observations in patients with endomyocardial fibrosis, confirmed by ventricular biopsy, are similar. ${ }^{21}$ In view of this suggestive evidence a more comprehensive study in man appeared justified.

If measurements of echo amplitude are to be compared between subjects some standard of comparison will be required. Many factors, including differences in path length, chest wall configuration, and cardiac orientation within the thorax militate against using an absolute external standard. We have, therefore, adopted a simpler expedient, that of using the echo from the parietal pericardium posterior to the left ventricular wall as an internal standard. This can be detected from the apex with an amplitude almost equal to that from the parasternal region, suggesting that echoes from it-as from other intracardiac structures-arise mainly by backscattering rather than by specular reflection. ${ }^{11}$ To validate its use in this way its collagen content was measured and found to be very high (approximately $50-65 \%$ of its dry weight). Variability was small not only between the anterior and posterior pericardium but also between patients. Although the collagen content of pericardium might theoretically be increased in disease, even total replacement by fibrosis could not increase its collagen content to more than $100 \%$, representing a factor of less than two, which is small compared with the range of collagen content observed in our patients. The relative uniformity of the constitution of parietal pericardium thus makes it suitable for use as an internal standard, allowing semiquantitative comparisons to be made between patients.

Estimates of myocardial collagen content in the present study are similar to those reported previously, using either histological ${ }^{-5}$ or biochemical methods. ${ }^{89}$ It became clear, however, that these criteria were not in complete agreement with one another in individual cases. These inconsistencies could have various causes. Histology depends on the tinctorial properties of the connective tissue elements, which are not well understood. Newly synthesised collagen may have less avidity for histological stains than older collagen, which is known to age chemically with formation of cross bridges linking lysine and hydroxylysine residues. Dissociation between histological and biochemical manifestations of collagen deposition may occur in acute disease; in bleomycin induced fibrosis, for example, increased collagen assessed biochemically is complete at two weeks, whereas histologically fibrosis is only beginning to appear at this stage. ${ }^{22}$ The method of chemical estimation used in the present study-that of detecting hydroxyproline containing proteins-was relatively non-specific. It is well recognised that within a single species there are at least five different collagen types, each with different physicochemical properties. ${ }^{23}$ Observations in experimental animals suggest that type III in particular is increased in left ventricular hypertrophy. ${ }^{24}$ In fibrotic lung disorders in man, there is biochemical evidence for a relatively greater increase in type III compared with type I collagen in the early stages of the disease. ${ }^{25}$ Detection of collagen by ultrasound depends on yet another physical property of collagen, its stiffness. There is no reason to suppose that measurements made on this basis would show complete agreement either with those based on chemical constitution or tinctorial properties any more than that the two latter should agree exactly with one another. The presence of increased amplitude of septal echoes suggests that there is no unique relation between collagen content and backscattering amplitude. The underlying cause of this was not clear. It did not appear to be a function of path length, since it was not shown by the posterior wall of the ventricle in infants when the ultrasound path length was much shorter than that to the septum in adults. Myocardial fibre arrangement in the septum is dominantly circumferential rather than oblique with varying fibre angle, as in the posterior wall, ${ }^{26}$ so that orientation of fibrosis might also have been more uniform in the septum. A similar mechanism has been invoked by 
Mimbs et $a l^{17}$ to explain increasing reflectivity after experimental myocardial infarction in the absence of pronounced increase in collagen content.

Yet other factors may well have aggravated lack of correspondence between the two methods. Fibrosis is often a patchy process, so that sampling errors are likely to have been present. Although samples for histological and chemical estimates came from the same anatomical segment, identical tissue could clearly not be used for both. Such errors are likely to have been particularly notable for histological estimates where the thickness of individual sections was less than 10 $\mu \mathrm{m}$, corresponding to less than $1 \mathrm{mg}$, while samples for biochemical analysis were approximately $5-30 \mathrm{mg}$ and those for pixel counting 500-1000 mg. Further errors are likely to have arisen when echocardiographic observations were correlated with necropsy findings, owing to failure to compare identical regions of myocardium. The feasibility of performing postmorten echocardiography was considered but rejected owing to the complexity of the changes in the acoustic properties of tissue after death. 2728 Minor differences between patients in the reflectivity of parietal pericardium would also have contributed to lack of complete agreement.

Finally, other disease processes might possibly have influenced regional echo amplitude. There is evidence that the acoustic properties of myocardium may be altered by experimental myocardial infarction. ${ }^{29}$ Although many of our patients were studied in the early postoperative period, there was no macroscopic or microscopic evidence of recent infarction at necropsy. The same applies to the suggestion that echo amplitude might have been increased by myocarditis, causing local inflammation or oedema. Despite these considerations, the correlation coefficient observed in the present study $\left(r^{2}=56 \%\right)$ shows that the dominant determinant of regional echo amplitude in the myocardium of the patients we studied was their collagen content assessed in terms of hydroxyproline concentration.

Our results clearly indicate the practical difficulties that will arise when tissue characterisation is attempted under clinical conditions. Tissue characterisation requires quantitative observation of returning echoes. ${ }^{30}$ In turn, this implies reproducible calibration of signals and standardisation of apparatus, which have not yet been achieved clinically. Secondly, the aspect of the tissue that it is proposed to "characterise" needs to be unambiguously defined. Fibrosis of myocardium may seem to be a well defined entity, and one that would be eminently accessible to such studies, but the disparity between histological and biochemical methods shows that this is not the case. If such difficulties arise with fibrosis the possibilities for confusion in defining processes such as "ischaemia" are clear, while an extensive search of published reports now attests to the difficulties of unambiguous measurement of "infarct size" under clinical conditions. A third factor is the rather poor reproducibility of measurements of echo amplitude. Clearly, no comparison can give closer results than that of the reproducibility of measurement of either of the variables involved. Although many of the determinants of echo amplitude were fixed between estimations such as machine gain, path length, and patient orientation, considerable variation still occurred owing to other, uncontrolled factors-such as motion of the heart with respiration or minor variation in transducer orientation or positioning of the area of interest leading to failure to sample identical areas of myocardium. Amplitude measurements are relatively simple to make compared with those related to phase, frequency dependence, or texture, which have been proposed as possible bases of tissue characterisation. ${ }^{31}$ Despite these difficulties, however, it does appear that in patients with chronic heart disease there is a semiquantitative relation between regional echo amplitude and myocardial collagen content. This can be shown in man by objective measurement of the median value of regional pixel intensity, using parietal pericardium as an internal standard and colour coded echocardiography as a visual prompt. Since myocardial fibrosis is increased in several common types of left ventricular disease, the possibility of making such measurements may well have clinical importance in documenting its presence and in assessing methods designed to prevent its appearance or even to cause its regression. The relatively simple techniques described here thus allow echocardiography to be used to gain information about the properties of the myocardium beyond simply its position and motion. Such studies may contribute clinically useful information in the future.

We acknowledge the assistance of Drs AJ Livett and RC Preston, Division of Radiation Science and Acoustics, National Physical Laboratory, Teddington, in calibrating the echocardiograph.

We acknowledge support from Corda and Special Cardiac Fund, Brompton Hospital.

\section{References}

1 Fuster V, Danielson MA, Robb RA, Broadbent JC, Brown AL Jr, Elveback LR. Quantitation of left ventricular myocardial fiber hypertrophy and interstitial tissue in human hearts with chronically increased volume and pressure overload. Circulation 1977; 55: 504-8.

2 Moore GW, Hutchins GM, Bulkley BH, Tseng JS, Ki PF. Constituents of the human ventricular myocardium: tissue hyperplasia accompanying muscular hypertrophy. Am Heart f 1980; 100: 610-6. 
3 Donaldson RM, Florio R, Rickards AF, et al. Irreversible morphological changes contributing to depressed cardiac function after surgery for chronic aortic regurgitation. Br Heart f 1982; 48: 589-97.

4 Oldershaw PJ, Brooksby IAB, Davies MJ, Coltart DJ, Jenkins BS, Webb-Peploe MM. Correlations of fibrosis in endomyocardial biopsies from patients with aortic valve disease. Br Heart $\mathcal{F}$ 1980; 44: 609-11.

5 Schwarz F, Flameng W, Schaper J, et al. Myocardial structure and function in patients with aortic valve disease and their relation to postoperative results. $A m \boldsymbol{F}$ Cardiol 1978; 41: 661-9.

6 Anderson KR, St John Sutton MG, Lie JT. Histopathological types of cardiac fibrosis in myocardial disease. F Pathol 1979; 128: 79-85.

7 Davies MJ, Fulton WFM, Robertson WB. The relation of coronary thrombosis to ischaemic myocardial necrosis. f Pathol 1979; 127: 99-110.

8 Caspari PG, Newcomb M, Gibson K, Harris P. Collagen in the normal and hypertrophied human ventricle. Cardiovasc Res 1977; 11: 554-8.

9 Frederiksen DW, Hoffnung JM, Frederiksen RT, Williams RB. The structural proteins of normal and diseased human myocardium. Circ Res 1978; 42: 459-66.

10 Shaw TRD, Logan-Sinclair RB, McAnulty RJ, et al. Relation between regional echo intensity and myocardial connective tissue in left ventricular disease. Fournal of Medical Science 1982; 10: 691-2.

11 Logan-Sinclair RB, Wong CM, Gibson DG. Clinical application of amplitude processing of echocardiographic images. Br Heart $₹$ 1981; 45: 621-7.

12 Bacon DR. Characteristics of a pvdf membrane hydrophone for use in the range 1-100 MHz. Transactions on Sonics and Ultrasonics 1982; 29: SU-18-25.

13 Edwards WD, Tajik AJ, Seward JB. Standardized nomenclature and anatomic basis for regional tomographic analysis of the heart. Mayo Clin Proc 1981; 56: 479-97.

14 Prockop DJ, Udenfriend S. A specific method for the analysis of hydroxyproline in tissues and urine. Anal Biochem 1960; 1: 228-39.

15 Laurent GJ, Cockerill P, McAnulty RJ, Hastings JRB. A simplified method for quantitation of the relative amounts of Type I and Type III collagen in small tissue samples. Anal Biochem 1981; 113: 301-12.

16 Fields S, Dunn F. Correlation of echographic visibility of tissue with biological composition and physiological state. F Acoust Soc Am 1973; 54: 809-12.

17 Mimbs JW, O'Donnell M, Bauwens D, Miller JW, Sobel $B E$. The dependence of ultrasonic attenuation and backscatter on collagen content in dog and rabbit hearts. Circ Res 1980; 47: 49-58.
18 Mimbs JW, O’Donnell M, Miller JG, Sobel BE. Detection of cardiomyopathic changes induced by doxorubicin based on quantitative analysis of ultrasonic backscatter. Am $\mathcal{F}$ Cardiol 1981; 47: 1056-60.

19 Rasmussen S, Corya BC, Feigenbaum H, Knoebel SB. Detection of myocardial scar tissue by $\mathrm{M}$-mode echocardiography. Circulation 1978; 57: 230-7.

20 Tanaka M, Terasawa H. Echocardiography: evaluation of the tissue character in myocardium. Fpn Circ $\mathcal{F}$ 1979; 43: 367-76.

21 Davies J, Gibson DG, Foale R, et al. Echocardiographic features of eosinophilic endomyocardial disease. Br Heart f 1982; 48: 434-40.

22 Laurent GJ, McAnulty RJ, Corrin B, Cockerill P. Biochemical and histological changes in pulmonary fibrosis induced in rabbits with intratracheal bleomycin. Eur $\mathcal{F}$ Clin Invest 1981; 11: 441-8.

23 Bornstein P, Sage H. Structurally distinct collagen types. Annu Rev Biochem 1980; 49: 957-1003.

24 Medugorac I, Jacob R. Characterisation of left ventricular collagen in the rat. Cardiovasc Res 1983; 17: 15-21.

25 Kirk JME, Heard B, Kerr I, Laurent GJ. Cryptogenic fibrosing alveolitis. Measurement of type I and III collagen in biopsy and postmortem lung samples, and relationship to clinical physiological and histological findings. Clin Sci 1983 (in press).

26 Greenbaum RA, Ho SY, Gibson DG, Becker AE, Anderson RH. Left ventricular fibre architecture in man. Br Heart f 1981; 45: 248-63.

27 Parry RJ, Chivers RC. Data of the velocity and attenuation of ultrasound in mammalian tissues. A survey. Washington: National Branch of Standards special publication 525, 1979: 343-57.

28 O'Donnell M, Mimbs JW, Sobel BE, Miller JG. Ultrasonic attenuation of myocardial tissue: dependence on time after excision and on temperature. $\mathcal{F}$ Acoust Soc Am 1977; 62: 1054-7.

29 Mimbs JW, Bauwens D, Cohen RD, O'Donnell M, Miller JG, Sobel BE. Effects of myocardial ischemia on quantitative ultrasonic backscatter and identification of responsible determinants. Circ Res 1981; 49: 89-96.

30 Chivers RC. Tissue characterization. Ultrasound Med Biol 1980; 7: 1-20.

31 Logan Sinclair RB, Oldershaw PJ, Gibson DG. Computing in echocardiography. Prog Cardiovasc Dis 1983 25: 465-86.

Requests for reprints to Dr D G Gibson, Brompton Hospital, Fulham Road, London SW3 6HP. 